\title{
Personalized subcutaneous administration of hepatitis B surface antibodies without nucleos(t)ide analogues is highly effective and reduces cost for hepatitis B prophylaxis after liver transplantation
}

Bielen Rob, $\mathrm{MD}^{1}$, Robaeys Geert, MD, $\mathrm{PhD}^{1,2}$, Sigrid Schelfhout ${ }^{3}$, Monbaliu Diethard, MD, $\mathrm{PhD}^{4}$, Van der Merwe Schalk MD, $\mathrm{PhD}^{3}$, Pirenne Jacques, $\mathrm{MD}, \mathrm{PhD}^{4}$, Nevens Frederik, MD, $\mathrm{PhD}^{3}$.

${ }^{1}$ Faculty of Medicine and Life Sciences, Hasselt University, Belgium.

${ }^{2}$ Department of Gastroenterology and Hepatology, Ziekenhuis Oost Limburg, 3600 Genk, Belgium.

${ }^{3}$ Department of Gastroenterology and Hepatology, University Hospitals KULeuven, Belgium.

${ }^{4}$ Department of Abdominal Transplant Surgery, University Hospitals KULeuven, Belgium. 


\section{ABSTRACT}

Background and Aims

Intravenous Hepatitis B Immunoglobulins (HBIG) in combination with nucleos(t)ide analogues (NAs) are the cornerstone of prophylaxis against Hepatitis B recurrence after liver transplantation (LT). Long-term use of IV HBIG has a high cost and the regular admission in the hospital is inconvenient. NAs alone does not prevent always HBsAg recurrence and can be nephrotoxic. SC HBIG can be self-administered. The optimal dose of SC HBIG without concomitant use of NAs has never been studied.

\section{Methods}

This is an investigator driven, prospective trial. All patients receiving IV HBIG were switched to SC HBIG (Zutectra ${ }^{\circledR}$ ) without NAs. The doses and interval of SC HBIG administration were aimed to keep HBsAg and HBV DNA undetectable. First dosage of Zutectra ${ }^{\circ}$ was based on the guidelines of the manufacturer $(<$ $75 \mathrm{~kg}$ : $500 \mathrm{IU} /$ week; $\geq 75 \mathrm{~kg}: 1.000 \mathrm{IU} /$ week). Thereafter, the titer of HBsAb was monitored regularly and if the titer was higher than the target levels at 2 successive occasions, a dose reduction was executed. In patients with low risk of recurrence (pts with undetectable HBV without antiviral therapy before LT, pts with acute liver failure and Delta hepatitis co-infected pts), the targeted titer was $\geq 100 \mathrm{IU} / \mathrm{I}$ and in the other patients $\geq 200 \mathrm{IU} / \mathrm{I}$. The tolerance of the patients (IV or SC) was assessed by a specific questionnaire.

Results

44 patients were included in this trial. One patient preferred to switch again to IV HBIG, all the others $(n=43)$ preferred SC HBIG, they did not report side effects and the compliance was $100 \%$. The mean time after LT was $9 \pm 6$ years. Mean follow up time was 2 years \pm 7 months. None of the patients had a relapse of HBsAg or HBV DNA. The mean HBsAb titer before the study was $332 \pm 173 \mathrm{IU} / \mathrm{I}$. The mean HBsAb titer 
at the end of the follow up period was $253 \pm 121 \mathrm{IU} / \mathrm{I}$ in the low risk group $(n=14)$ and $281 \pm 91 \mathrm{IU} / \mathrm{I}$ in the high risk group ( $n=21)$. In 76\% ( $n=33$ ) doses reductions were possible. The total combined dose at the start was reduced from $118.000 \mathrm{IU} /$ month to $68.135 \mathrm{IU} / \mathrm{month}$. The median frequency of injections reduced from $1 / w$ (range $2 / w-1 / w)$ to $1 / 2 w$ (range $2 / w-1 / 3 w)$.

Conclusions

All except one patient preferred subcutaneous HBIG. SC HBIG without NAs had a $100 \%$ success rate in the long-term prevention of HBsAg and HBV DNA reappearance. Doses adaptation based on pre LT risk factors for HBV recurrence resulted in the vast majority of the pts in reduction of doses and/or prolongation of the interval and together with the self-administration and the no use of NAs induced a significant reduction of cost. 